\title{
Predictors of HIV Testing Positive Result Among Pregnant Women Attending Prenatal Clinics in the Democratic Republic of Congo
}

\author{
Jack Hyyombo Tambwe Kokolomami*, Patrick Kalambayi Kayembe \\ Department of Epidemiology and Biostatistics, Kinshasa School of Public Health, University of Kinshasa, Kinshasa, the Democratic \\ Republic of Congo
}

Email address:

pajack70@yahoo.fr (J. H. T. Kokolomami)

${ }^{*}$ Corresponding author

To cite this article:

Jack Hyyombo Tambwe Kokolomami, Patrick Kalambayi Kayembe. Predictors of HIV Testing Positive Result Among Pregnant Women Attending Prenatal Clinics in the Democratic Republic of Congo. Central African Journal of Public Health. Vol. 4, No. 4, 2018 , pp. 125-130. doi: $10.11648 /$ j.cajph.20180404.15

Received: August 9, 2018; Accepted: August 22, 2018; Published: September 27, 2018

\begin{abstract}
Since it began in 2003, HIV/AIDS surveillance in Democratic Republic of the Congo has been mainly carried out among pregnant women attending antenatal clinics. In addition to screening for syphilis and HIV, some demographic, behavioral, and obstetrical data are also collected. This work aimed to identify the factors associated with a positive HIV test result from the demographic and medico-obstetrical data collected at the time of surveillance-based HIV testing among pregnant women attending sentinel sites in DRC. The HIV surveillance database among pregnant women was obtained from the National AIDS Control Program of DRC. The association between the HIV test result and the factors studied was assessed by the adjusted Odds Ratio (AOR), through a logistic regression of HIV serology on these potential factors. From 2003 to 2013, 109,553 pregnant women were screened for HIV in the DRC surveillance sites. The regression of the HIV test result on the possible factors showed among others that: (i) primary or secondary education was associated with a higher odd of test positivity (AOR: 1.51, 95\% CI: $1.13-2.03$ and $1.41,95 \%$ CI $1.05-1.89$ respectively) compared to the higher/university level of education; (ii) the sexual debut before age 15 (AOR: 1.33, 95\% CI: 1.21 - 1.46) or between 15 and 17 years of age (AOR: $1.18,95 \%$ CI: $1.09-1.28$ ) was associated with a positive test result in comparison to age 18 and older; and (iii) the presence of an active syphilis infection was associated with a twofold increase in HIV positive test result odd (AOR: 2.01, 95\% CI: 1, 772.29). Primary prevention efforts against HIV infection in DRC should include delaying sexual debut, encouraging complete formal education of women, and preventing and adequately managing sexually transmissible infections.
\end{abstract}

Keywords: HIV Test Positive Result, Pregnant Women, DRC, Predictors

\section{Introduction}

In the DRC, data on the HIV / AIDS epidemic across the country are rather limited. The most regular are those obtained from successive sentinel surveillance activities among pregnant women attending Ante-Natal Clinics (ANC). Other sources of data include: the Demographic and Health Surveys with HIV testing component (DHS +, 2007 and 2013), some reports from field interventions and control programs and projects, and national science days on HIV/ AIDS in the DRC, which allow researchers and stakeholders to actively discuss various research findings on the subject.
After fifteen years of continuous sentinel surveillance, current thinking is to use more and more programmatic data, especially PMTCT sites data, for surveillance purposes. The difficulty in doing this is that the screening test is performed on an agreed basis on PMTCT settings, whereas it is anonymous and unrelated on surveillance sites. The requirement of consent may exclude some women on PMTCT sites, and selectively those who fear a positive test result. The prevalence of HIV on PMTCT sites would therefore be lower than that of sentinel sites, or that of the general population. This is what other authors have already found elsewhere [1]. In addition to some problems in data 
reporting, PMTCT sites regularly experience breaks in inputs or other resources, leading them to discontinue screening, or at least to rationalize it, targeting women considered to be at higher risk of HIV infection [2]. Hence, one of the pillars of the fight against HIV/AIDS, and particularly the elimination of mother-to-child transmission of HIV in the DRC, is the prevention of HIV in future birth mothers. Around the world, researchers have studied the factors associated with HIV infection, in the general population as with pregnant women, in order to contribute to a prevention battle based on evidence [3-4]. This research was carried out to identify, at the individual level, the factors associated with HIV positive serology in pregnant women attending ANC in the DRC, in order to better guide prevention interventions and, on the other hand, to focus screening programs, in a context of insufficient resources [5].

\section{Methodology}

Databases of HIV sentinel surveillance on pregnant women attending ANC in the DRC from 2003 to 2013 were used for this research. Annually, teams of central supervisors are sent to sentinel HIV surveillance sites and, with provincial support, oversee the implementation of surveillance activities. This ensures compliance with a single, validated protocol, which makes the data comparable. From 2003 to 2013, seven annual rounds collected the information, including:

- Sociodemographic and behavioral data: on the basis of a short two-page questionnaire, data such as age of the woman, marital status, education level, age at sexual debut, age of current sexual partner, number of pregnancies already carried, number of blood transfusions received, are collected.

- Biological data: in accordance with standardized procedures, aliquots obtained from the systematic samples taken for syphilis screening are used, separated from any information that could link them to the person, and then tested for HIV algorithm. The positive samples and $10 \%$ of the negative samples are confirmed at the national reference laboratory on HIV and STI in Kinshasa. This disposal guarantees the validity of the diagnosis. As recently as 2013, protocol modifications have been made to match the surveillance sites with those of PMTCT and to allow access to the results of the test by participants. As the Protocol changed, the information collected that year forward could be different from that collected previously. To obtain comparable information for 2013, in order to properly merge this year's data to those previous, the 17,211 blood samples collected that year were re-screened for HIV in accordance with the protocol and algorithm used the years before (2003 to 2011).

Once a single database had been established, it was processed to handle missing data, code text responses, and turn some variables into desired categories.

A binary logistic regression of the HIV test result on a group of factors potentially determining the serology was performed. The possible association between HIV serology and any of the factors collected on the record was assessed by the adjusted Odds Ratio and its 95\% confidence interval. The NCSS software (version 11) and the DESCRIBE program (version 3.18) of the WINPEPI software were used for these analyzes. Moreover, some graphs were formulated on Microsoft Excel.

\section{Results}

From 2003 to 2013, a total of 109,553 pregnant women were tested for HIV at the sentinel surveillance sites in the DRC. Table 1 shows the principal sociodemographic characteristics of those pregnant women.

Table 1. Sociodemographic characteristics of pregnant women screened for HIV in sentinel surveillance sites in DRC from 2003 to 2013 , based on the HIV test result.

\begin{tabular}{|c|c|c|c|c|}
\hline Characteristic & Modalities & Total screened & HIV Positives & $(\%)$ \\
\hline \multirow{4}{*}{ Age } & Adolescent (younger than 18 years old) & 9538 & 284 & $3,0 \%$ \\
\hline & Young adult (18 to 24 years old) & 44196 & 1544 & $3,5 \%$ \\
\hline & Adult ( 25 years and older) & 55725 & 2303 & $4,1 \%$ \\
\hline & Total & 109459 & 4131 & $3,8 \%$ \\
\hline \multirow{5}{*}{ Education } & No formal instruction & 12776 & 379 & $3,0 \%$ \\
\hline & Primary level instruction & 45965 & 1753 & $3,8 \%$ \\
\hline & Secondary level instruction & 48601 & 1938 & $4,0 \%$ \\
\hline & Higher/University level instruction & 1811 & 49 & $2,7 \%$ \\
\hline & Total & 109153 & 4119 & $3,8 \%$ \\
\hline \multirow{5}{*}{ Occupation } & Without remuneration & 67794 & 2547 & $3,8 \%$ \\
\hline & Self-employed/entrepreneur & 35750 & 1378 & $3,9 \%$ \\
\hline & Employee & 3654 & 149 & $4,1 \%$ \\
\hline & Small/micro commercial enterprises & 2355 & 59 & $2,5 \%$ \\
\hline & Total & 109553 & 4133 & $3,8 \%$ \\
\hline \multirow{4}{*}{ Marital status } & Single (never married) & 6185 & 223 & $3,6 \%$ \\
\hline & Currently married (cohabiting) & 102051 & 3847 & $3,8 \%$ \\
\hline & Previously married (Separated, divorced, widowed) & 1317 & 63 & $4,8 \%$ \\
\hline & Total & 109553 & 4133 & $3,8 \%$ \\
\hline \multirow{4}{*}{ Age at sexual debut } & Before age 15 & 22427 & 933 & $4,2 \%$ \\
\hline & At 15 to 17 years & 57771 & 2198 & $3,8 \%$ \\
\hline & At 18 years and older & 29355 & 1002 & $3,4 \%$ \\
\hline & Total & 109553 & 4133 & $3,8 \%$ \\
\hline
\end{tabular}


Table 1 shows that: almost $10 \%$ of pregnant women tested for HIV in the DRC ANC from 2003 to 2013 were younger than 18 years of age; less than $2 \%$ of these women had reached the highest level of education; and that more than $90 \%$ of them cohabited with a male partner. As shown in
Figure 1, the average age at first sexual intercourse is younger for HIV-positive women, and even for syphilispositive, than negative ones. Considering the living environment, this age is younger for women residing in rural areas compared with those living in cities.

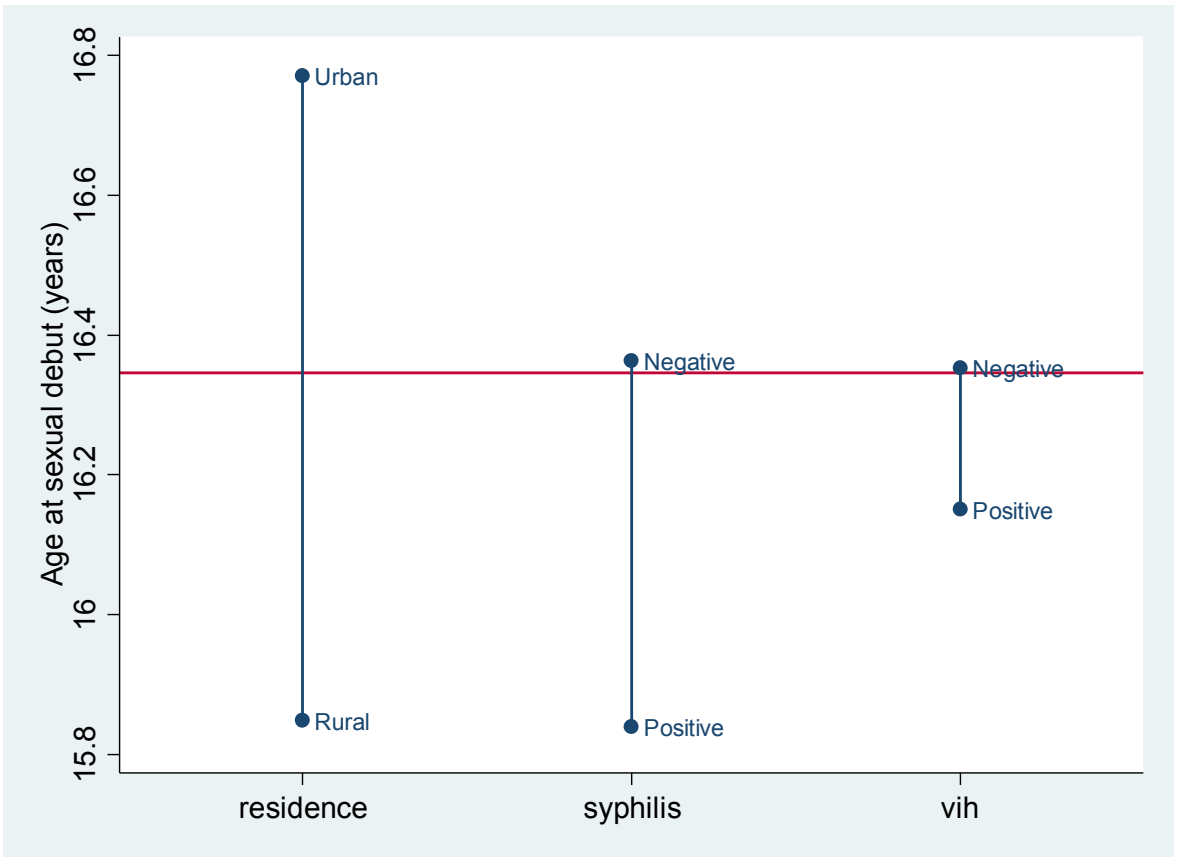

Figure 1. Mean age at sexual debut of pregnant women attending ANC services in the DRC, based on HIV and Syphilis serology, and living environment.

A binary logistic regression of HIV test results on the few potentially determining factors collected during the prescreening interview was performed. Only variables that were found to be associated with HIV serology on the basis of a bivariate analysis were introduced into the logistic model. For some of these factors (such as blood transfusion history, occupation, or marital status) the regression model did not detect any association with HIV serology. Table 2 depicts the results of the factors observed to be associated with HIV serology.

Table 2. Some determinants of HIV infection among pregnant women attending the ANC in the DRC.

\begin{tabular}{|c|c|c|c|c|c|}
\hline & & \multirow{2}{*}{ p (Wald test) } & \multirow{2}{*}{$\mathbf{O R}$} & \multicolumn{2}{|c|}{$95 \%$ CI of OR } \\
\hline & & & & Lower & Upper \\
\hline \multirow{3}{*}{ Age $(p<0.001)$} & Adolescent (less than 18 years old) & & 1 & & \\
\hline & Young (Age 18 to 24 years) & .001 & 1.26 & 1.10 & 1.43 \\
\hline & Adult (Age 25 or older) & .000 & 1.54 & 1.35 & 1.76 \\
\hline \multirow{3}{*}{ Education $(\mathrm{p}<0.001)$} & Superior/Higher/University level instruction & & 1 & & \\
\hline & Secondary level instruction & .006 & 1.51 & 1.13 & 2.03 \\
\hline & Primary level instruction & .024 & 1.41 & 1.05 & 1.89 \\
\hline \multirow{3}{*}{$\begin{array}{l}\text { Age at sexual debut } \\
(p<0.001)\end{array}$} & Before age 15 & & 1 & & \\
\hline & At 15 to 17 years & .000 & 1.18 & 1.09 & 1.28 \\
\hline & At 18 years and beyond & .000 & 1.33 & 1.21 & 1.46 \\
\hline \multirow{2}{*}{$\begin{array}{l}\text { Syphilis } \\
(\mathrm{p}<0.001)\end{array}$} & Negative & & 1 & & \\
\hline & Positive & .000 & 2.01 & 1.77 & 2.29 \\
\hline
\end{tabular}

The data in this table show that:

- Considering the age of the pregnant women: HIVpositive odd for pregnant women was $26 \%$ and $54 \%$ higher for young adults (18-24 years) and adults (25 years and older) respectively, compared with that for adolescent girls (15-17 years of age). A woman's age is therefore a factor associated with the positivity result of an HIV test among pregnant women in the DRC. The older the age is; the higher the HIV-positive odd.

- Women's level of education is another factor associated with a positive HIV test result among pregnant women. Here the ratio is the opposite: the more highly educated the woman, the lower the positive odd. Women without formal education, with only primary, and secondary education, experienced a $4 \%, 41 \%$, and $51 \%$ increase in positive status, respectively, in comparison with those who reached higher or 
university education.

- In terms of age at first sexual intercourse: compared with women whose sexual debut was at age 18 or later, the HIVpositive odds among women who had their first sexual intercourse between the ages of 15 and 17, and those before 15 years, are increased by $18 \%$ and $33 \%$ respectively.

- In the end, pregnant women with a positive syphilis test result, had an HIV positive test odd double that of pregnant women with a negative syphilis test result.

\section{Discussion}

This research explored sentinel HIV surveillance data among pregnant women in the DRC and sought to identify factors associated with the positivity of the HIV test among these women. It found that the age of a woman, her level of education, her age at first intercourse, and the presence of a sexually transmitted infection (in this case syphilis) are factors associated with HIV infection; while her profession, marital status, and the fact of having=undergone a transfusion, are not associated.

Age is known factor associated with HIV infection. In this study, the age of 25 years and older is more associated with this infection, and less associated with under- 25 years olds. In a context of predominantly sexual transmission of HIV, as in the DRC, sexual experience and the number of different sexual partners increases with age, which could explain this difference [6]. Exposure to other routes of contamination is also all the more intense as age advances. In Kenya, Johnson and colleagues found that the 25-29 age group for women, and the 35 to 44 age group for men, were associated with a high positive HIV test score [7]. In India, G. Alvarez-Uria and his team found that, in rural areas, the age range of 25 to 45 year olds was associated with a high HIV positive status [8].

Low educational attainment is also a known risk factor for HIV infection [4]. The acquisition of certain skills necessary for preventive action depends on education. Our study has shown that the higher the level of education, the lower is the odd of HIV positivity. Education is therefore a significant protective factor against HIV infection. Other researchers have described this elsewhere. In point is a Tanzanian study in which E. Mmboma describes education as being associated with HIV infection in rural settings, and mostly educated women as having a lower likelihood of infection [9]. Another Tanzanian study in point conducted by G. Todd and his team found that people who have at least completed primary education have a lower probability of contracting HIV than those who have not received any formal education [10]. In one more study in point, Alvarez-Uria and his team made the same observation of men as aforementioned [8]; and likewise $\mathrm{U}$. Thamattoor and his collaborators of pregnant women attending ANC [4].

Early sexual debut, along with the multiplicity of sexual partners, increases the risk of HIV infection [11, 12]. Our study showed that the more the first sexual intercourse was delayed, the lower the HIV-positive odd, so that the group of women who had had sexual intercourse before 15 years of age had an HIV-positive odd increased by 33\% compared with the group that started at age 18 or older. Other researchers also made these findings. For example, J. Kembo found that in Zimbabwe young people whose first sexual intercourse occurred before 14 years of age were significantly more at risk of contracting HIV infection (2.696 times higher than their counterparts for whom their first sexual experience took place at 20-24 years of age) [13]. Moreover, in Zambia the decline in the proportion of young people beginning the sexual experience before age 15 was one of the factors contributing to the significant decline in HIV prevalence among young women aged 15-24 [14]. In a South African study, Wand and Ramjee found that women whose age at first intercourse was 15 years of age or younger had a higher incidence of HIV than women who started later [12]. Early sexual intercourse may itself be due to acts of violence (such as rape), or to early and/or arranged marriages. Even in this context, it remains a major risk factor for HIV infection, as noted by L. Ackerman and GW de Klerk [15]. In a Chinese study, R. Xu and colleagues found that even among men who had sex with men, the HIVpositive odd was higher among those who had started these relationships earlier [16]. The reason for this association between early sexual intercourse and a positive HIV test result is that; the sooner the sexual experience begins, the longer it will be in a lifetime, the greater the likelihood of a multi-partnership sexuality, and also the probability that at least one of these sexual partners is infected. The biological fragility during adolescence has also to be mentioned [17]. Research has already shown that this biological fragility exposes young girls to HIV infection, more than young boys [18].

The presence, or even the history, of a sexually transmitted infection is known to be a major risk factor for HIV infection. For some STIs, the interaction with HIV infection is bi-directional: STI increases the incidence of HIV infection, and the latter affects the prognosis of the STI [19]. The present study shows that the odd of positive HIV test result is twice as high among women with syphilis compared with those, without. Moreover, Korenromp and collaborators found the same association in Tanzania [20]. In South Africa, it was found a HIV-positive odds ratio of 1.5 between the two groups [21]. In the United States, an HIV-positive test result odds ratio of 5.0 and 4.2 was found respectively in women and men with syphilis compared with those, without. STIs also influence the natural evolution of HIV infection, and the viral load [22]. The high prevalence of syphilis among pregnant women in our series $(4.4 \%$ in 2013$)$ is therefore both a risk factor for HIV infection and also a factor of poor prognosis in its evolution.

\section{Limitation of Study}

The main limitation of this research is the use of data collected by different actors over a decade. We therefore assumed the results of the HIV and syphilis tests reported by 
the successive teams. However, the control measures and even the quality assurance and compliance with the monitoring protocol put in place reassure the validity of the data. Another limitation could have been the fact that the monitoring protocol was modified after 2011. This difficulty was solved as we, the researchers, used to re-test all the samples of 2013 on the basis of the previous algorithm.

\section{Conclusion}

In the DRC, the typical HIV-positive pregnant woman is a woman aged 25 years and older, having stopped education at the secondary school level, having had sexual dubut before age 15 , and carrying a sexually transmitted infection. Primary prevention efforts against HIV infection should include delaying first sexual intercourse, encouraging complete formal education of women, and preventing and adequately managing sexually transmissible infections among sexually active young people.

\section{References}

[1] Pereira GFM, Sabidó M, Caruso A, Benzaken AS. Transitioning from antenatal surveillance surveys to routine HIV testing: a turning point in the mother-to-child transmission prevention programme for HIV surveillance in Brazil. BMC Infect Dis [Internet]. BMC Infectious Diseases; 2017;17(1):469. Available from:

$\mathrm{http}: / /$ bmcinfectdis.biomedcentral.com/articles/10.1186/s1287 9-017-2540-4

[2] Young PW, Mahomed M, Horth RZ, Shiraishi RW, Jani I V. Routine data from prevention of mother-to-child transmission (PMTCT) HIV testing not yet ready for HIV surveillance in Mozambique: a retrospective analysis of matched test results. BMC Infect Dis [Internet]. BMC Infectious Diseases; 2013;13(1):96. Available from: http://bmcinfectdis.biomedcentral.com/articles/10.1186/14712334-13-96

[3] Toska E, Gittings L, Hodes R, Cluver LD, Town C, Cape W, et al. Resourcing resilience: social protection for HIV prevention amongst children and adolescents in Eastern and Southern Africa. 2017;15(2):123-40

[4] Thamattoor U, Thomas T, Banandur P, Rajaram S, Duchesne T, Abdous B, et al. Multilevel Analysis of the Predictors of HIV Prevalence among Pregnant Women Enrolled in Annual HIV Sentinel Surveillance in Four States in Southern India. $2015 ; 1-11$

[5] V ALRDV. Francisco I Bastos VI HIV testing among pregnant women in Brazil : rates and predictors Testagem anti-HIV em mulheres grávidas no Brasil: taxas e preditores. 2008;42(5):859-67

[6] Mohamed BA, Mahfouz MS. Factors Associated with HIV / AIDS in Sudan. Biomed Res Int. 2013;1(2013):1-6

[7] Johnson K, Way A. Risk factors for HIV infection in a national adult population: evidence from the 2003 Kenya Demographic and Health Survey. J Acquir Immune Defic Syndr. 2006;42(5):627-36
[8] Alvarez-uria G, Midde M, Naik PK. Socio-demographic risk factors associated with HIV infection in patients seeking medical advice in a rural hospital of India $m$ er us e on ly om $\mathrm{m}$ er ci al us e on. J Public health Res. 2012;1:e16

[9] Mmbaga EJ. HIV prevalence and associated risk factors: Analysis of change over time in mainland Tanzania. United States Agency Int Dev [Internet]. 2013;18(February):1-44. Available from:

http://dhsprogram.com/pubs/pdf/WP85/WP85.pdf

[10] Todd J, Grosskurth H, Changalucha J, Obasi A, Mosha F, Balira R, et al. Risk factors influencing HIV infection incidence in a rural African population: a nested case-control study. J Infect Dis [Internet]. 2006;193(3):458-66. Available from:

http://www.ncbi.nlm.nih.gov/entrez/query.fcgi?cmd=Retrieve $\& \mathrm{db}=$ PubMed\&dopt $=$ Citation\&list_uids $=16388496$

[11] Stöckl H, Kalra N, Jacobi J, Watts C. Is Early Sexual Debut a Risk Factor for HIV Infection Among Women in Sub-Saharan Africa? A Systematic Review. Am J Reprod Immunol [Internet]. 2013 Feb 1 [cited 2017 Sep 16];69(s1):27-40. Available from: http://doi.wiley.com/10.1111/aji.12043

[12] Wand H, Ramjee G. The relationship between age of coital debut and HIV seroprevalence among women in Durban, South Africa: a cohort study. BMJ Open [Internet]. 2012;2:e000285. Available from: http://www.pubmedcentral.nih.gov/articlerender.fcgi?artid=32 $53418 \&$ tool $=$ pmcentrez\&rendertype $=$ abstract

[13] Kembo J. Risk factors associated with HIV infection among young persons aged 15-24 years: evidence from an in-depth analysis of the 2005-06 Zimbabwe Demographic and Health Survey. SAHARA J [Internet]. 2012;9(2):54-63. Available from: http://www.ncbi.nlm.nih.gov/pubmed/23237040

[14] Kembo J. Changes in sexual behaviour and practice and HIV prevalence indicators among young people aged $15-24$ years in Zambia: An in-depth analysis of the $2001-2002$ and 2007 Zambia Demographic and Health Surveys analysis of the 2001 - 2002 and 2007 Zambia Dem. J Soc Asp HIV/AIDS [Internet]. Taylor \& Francis; 2014;0(0):1-13. Available from: http://dx.doi.org/10.1080/17290376.2014.903620

[15] Simbayi LC, Kalichman SC, Jooste S, Cherry C, Mfecane S, Cain D. Risk factors for HIV-AIDS among youth in Cape Town, South Africa. AIDS Behav. 2005;9(1):53-61

[16] Xu R, Dai W, Zhao G, Tu D, Yang L, Wang F, et al. Early Sexual Debut and HIV Infection among Men Who Have Sex with Men in Shenzhen, China. 2016;2016

[17] Kalra N, Jacobi J, Watts C. Is Early Sexual Debut a Risk Factor for HIV Infection Among Women in Sub-Saharan Africa? A Systematic Review. 2013;27-40

[18] Blignaut RJ, Jacobs J, Vergnani T. Trends in HIV risk behaviour of incoming first-year students at a South African university: 2007 - 2012. Taylor \& Francis; 2015;376(September 2017):2007-12

[19] Wilton J. STIs : What role do they play in HIV transmission? 2012

[20] Korenromp EL, White RG, Orroth KK, Bakker R, Kamali A, Serwadda D, et al. Determinants of the impact of sexually transmitted infection treatment on prevention of HIV infection: a synthesis of evidence from the Mwanza, Rakai, and Masaka intervention trials. J Inf Dis. 2005;191 Suppl(Suppl 1):S168-78 
[21] Jemmott JB, Jemmott LS, O’Leary A, Ngwane Z, Icard LD, Heeren GA, et al. Prevalence of and Factors Associated with Sexually Transmitted Infections amongst Patients Attending a Primary Healthcare Facility in Mthatha, Eastern Cape. Am J Public Health [Internet]. 2014;1(3):317-22. Available from: http://dx.doi.org/10.1016/j.contraception.2009.06.007\%5Cnhtt p://dx.doi.org/10.1016/j.srhc.2010.08.006\%5Cnhttp://dx.doi.o rg/10.1016/j.srhc.2010.03.001\%5Cn\%22http://dx.doi.org/10.1 007/s12119-016-9360-3
[22] Champredon D, Bellan SE, Delva W, Hunt S, Shi C-F, Smieja $\mathrm{M}$, et al. The effect of sexually transmitted co-infections on HIV viral load amongst individuals on antiretroviral therapy: a systematic review and meta-analysis. BMC Infect Dis [Internet]. BMC Infectious Diseases; 2015;15:249. Available from:

http://www.pubmedcentral.nih.gov/articlerender.fcgi?artid=44 86691\&tool=pmcentrez\&rendertype $=$ abstract 